\title{
DETERMINATION OF STRESS IN SPREAD FOUNDATION SUBSOIL BY VARIOUS APPROACHES
}

\author{
Giang NGUYEN ${ }^{1,2, *}$
}

${ }^{1}$ Department of Geotechnics, Faculty of Civil Engineering, University of Žilina, Univerzitná 8215/1, 01026 Žilina.

2 Institute of Textile Engineering and Polymer Materials, Faculty of Materials and Environment Sciences, University of Bielsko - Biela, Poland.

* corresponding author: giang.nguyen@fstav.uniza.sk, +421-4-1513 5759; gnguyen@ath.bielsko.pl, +48-33-827 9175 .

\begin{abstract}
The paper deals with the determination of stress induced in spread foundation subsoil by Slovak Technical Standard STN 73 1001:2010 (STN) and Polish Standard PN-81/B-03020 (PN). While STN does not consider with stress state in subsoil just after carrying out excavation and the stress is determined from overloading only, PN considers with stress states after carrying out excavation and follows loading. Differences are also in determination of foundation stiffness and taking into account soil structural strength. It will be shown that different approaches make difference in the value of additional stress up to $18.6 \%$.
\end{abstract}

\author{
Keywords: \\ Subsoil; \\ Foundation stiffness; \\ Stress from external loading; \\ Soil rebound; \\ Soil structural strength.
}

\section{Introduction}

An important problem of soil mechanics practice is the prediction of the settlements of a structure built on the soil. For a homogeneous isotropic linear elastic material the deformations could be calculated using the theory of elasticity. That is a completely consistent theory, leading to expressions for the stresses and the displacements. However, solutions are available only for a half space and a half plane, not for a layered material (at least not in closed form). Moreover, soils exhibit non-linear properties (such as a stiffness increasing with the actual stress), often have anisotropic properties, and in many cases the soil consists of layers of different properties. For such materials the description of the material properties is already a complex problem, let alone the analysis of stresses and deformations. For these reasons, an approximation is often used, based on a semielastic analysis. In this approximation, it is proposed that the vertical stresses in the soil can be approximated by the stresses that can be calculated from linear elastic theory. On the basis of these stresses, the deformations are then determined, using the best available description of the relation between stress and strain, which may be non-linear. If the soil is layered, the deformations of each layer are calculated using its own properties, and then the surface displacements are determined by a summation of the deformations of all layers. In this way, the different properties of the layers can be taken into account, including a possible increase of the stiffness with depth. The procedure is not completely consistent, because in a soil consisting of layers of different stiffness, the stress distribution will not be the same as in a homogeneous linear elastic material. A partial justification may be that the stresses following from an elastic computation at least satisfy the equilibrium conditions. Also it has been found, by comparison of solutions for layered materials with the solution for a homogeneous material, that the distribution of the vertical stress $\sigma_{z}$ is not very sensitive to the material properties, provided that the differences in material properties are not very large [1].

When designing spread foundation, one needs to determine stresses induced in subsoil, which are necessary for its settlement calculation and after that structure verification for serviceability limit states. The change of stress state depends on many factors, excepting above mentioned, mainly on the depth of excavation, on the value of structure load and foundation stiffness. Before excavation is carried out, the subsoil is loading by geostatic stress. Since the effective stress causes soil deformation; in this case, the effective geostatic stress is determined. After excavation is carried out, the geostatic stress is decreased by soil removing. After starting spread foundation construction, stress from external loading will induce in subsoil. The stress from external loading will be higher and 
higher when construction is in progress. So there is a question how to take into account influence of soil removing and loading again.

There are also questions how to take into account e. g. influence of foundation depth, influence of practically incompressible layer being in small depth under foundation in practice design etc. The authors in [2] introduce a diagram, where one can see influence of foundation depth on the values of vertical stress in subsoil under strip foundation. They state that assumptions of theoretical solutions are unrealistic and values of vertical stress calculated by STN [3] are applicable. Similarly, they state that the conformity of the values of vertical stress, taking into account influence of practically incompressible layer being in small depth under foundation, calculated by STN, with such one, calculated using finite element method, is acceptable. On the other hand, e. g. PN [4] does not take into account influence of foundation depth and influence of practically incompressible layer being in small depth under foundation by such way as STN does. Then one can expect that values of vertical stress, calculated by the STN and PN will be different. In the following, we will introduce the determination of such stresses by the STN and the PN.

\section{Determination of stress in subsoil by the STN}

The STN prescribes the determination of the stiffness of system "subsoil - spread foundation“, by which the positions, in which stress will be calculated, will be defined. The stiffness of system „subsoil - spread foundation“ is roughly defined for rectangle foundation by the formula:

$k=\frac{E}{E_{\text {daff }}}\left(\frac{\mathrm{t}}{\mathrm{L}}\right)^{\mathrm{a}}$

where $E$ is the elastic modulus of the material of foundation structure (e.g. in MPa), $\overline{E_{d e f}}$ is the average deformation modulus of subsoil to the depth of deformation zone (e. g. in MPa), $t$ is the thickness of foundation structure (e.g. in $\mathrm{m}$ ), $l$ is the size of foundation structure in the direction in which the stiffness will be determined (e.g. in $\mathrm{m}$ ).

The average deformation modulus of subsoil to the depth of deformation zone can be calculated using e. g. the formula:

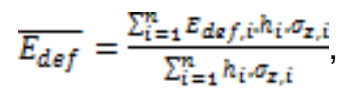

where $E_{\text {def } i \mathrm{i}}$ is the deformation modulus of i-layer in range of deformation zone (e.g. in MPa), $h_{\mathrm{i}}$ is the thickness of i-layer in range of deformation zone (e.g. in $\mathrm{m}$ ), $\sigma_{z, i}$ is the average value of vertical stress in i-layer from structure (e.g. in $\mathrm{MPa}$ ).

If $k<1$, the foundation structure is flexible and it is possible to calculate the settlement from the vertical stress, calculated by the theory of elastic half-space (attachment A in the STN). If $k>1$, the foundation structure is considered to be stiff and the settlement will be determined from the vertical stress under the characteristic point, where the theoretical settlement of flexible and stiff foundation is the same. The rectangle foundation has characteristic point with the coordinates $0.37 \mathrm{~B}$ and $0.37 \mathrm{~L}$ (from the foundation centre, where $B$ and $L$ are foundation width and length); for the circle foundation, the characteristic points lay in the circle with the radius $0.85 r$ (where $r$ is the foundation radius). The characteristic point is not defined for other foundation shape. In more important cases, the determining stress and common deformation of structure and foundation by interaction methods is recommended.

When calculating the effect of load by structure to evaluate the second group of limit states, it is possible, for calculation of settlement of stiff foundation, to suppose the uniform distribution of contact stress:

$\sigma_{d s}=\frac{V_{d s}}{A}$,

where $\sigma_{d g}$ is the service design contact stress in foundation base (e.g. in $\mathrm{kPa}$ ), $V_{d g}$ is the service design vertical force (e.g. in $\mathrm{kN}$ ), $A$ is the pressed part of foundation base (e.g. in $\mathrm{m}^{2}$ ).

In the case of off-centre position of the force $V_{d s}$, if it is necessary, calculation of inclination using moment in foundation base or edge stresses will be also carried out. The largest eccentricity 
$e_{\max }$ is not allowed to exceed $1 / 3$ foundation size in the direction of acting eccentricity. For the case of eccentricity, where the force is acting outside of the foundation cross section kernel, the contact stress in foundation base will be determined without tension. The circle and regular polygon foundation can be replaced by square with the same area. For the rectangle foundation and eccentric loading:

$\left(\frac{e_{b}}{b}\right)^{2}+\left(\frac{e_{l}}{d}\right)^{2} \leq\left(\frac{1}{a}\right)^{2}$.

For the flexible foundation, it is recommended to carry out the settlement calculation, foundation base deformation and contacting stress considering mutual influence of foundation and subsoil.

For the calculation concerning the second group of limit states, it is possible approximately to include the influence of foundation depth on the value of vertical stress $\sigma_{z}$ in the depth $z$ under foundation base by this way that it can be calculated with substituted depth $z_{r 1}$ by the formula:

$z_{r 1}=\chi_{1} \cdot z$

Then the depth $z_{r 1}$ will be used instead of the depth $z$ when calculating the vertical stress $\sigma_{z}$. The influence of practically incompressible layer being in small depth $z_{i c}$ under foundation is included into calculation of vertical stress $\sigma_{z}$ by such way that instead of the depth $z$ under foundation we will use the depth $z_{r 2}$ which can be determined as:

$z_{r 2}=\chi_{2} \times z$

In the case of both influences (influence of foundation depth and influence of practically incompressible layer) will be presented when calculating vertical stress $\sigma_{z}$, we will use substituted depth $z_{r a}$ which can be determined as:

$z_{\gamma a}=\chi_{1} \cdot \chi_{2}, z$

The values of $\chi_{1}$ can be determined using formula (for pad foundation):

$\chi_{1}=1+0.35 \operatorname{arctg}\left(1.55 \cdot \frac{D}{z}\right)$,

where $D(\mathrm{~m})$ is the depth of foundation and $z(\mathrm{~m})$ is the depth under foundation base in which induced stress will be determined.

The values of $\chi_{2}$ can be determined using formula (for pad and strip foundation):

$\chi_{2}=1-\exp \left(\frac{z_{\text {ir }}}{z} \ln 0.25+\ln 0.8\right)$,

where $z_{\text {ic }}(\mathrm{m})$ is the distance between foundation base and soil stiff layer surface; $z(\mathrm{~m})$ is the depth under foundation base in which induced stress will be determined.

The vertical stress $\sigma_{z}$ in the depth $z$ under the corner of the rectangle foundation with the width $B$ and length $L$, loaded by uniform loading $f$ can be calculated using formula:

$\sigma=\frac{f}{2 \cdot \pi} \cdot\left[\operatorname{arctg} \frac{L \cdot B}{Z \cdot \sqrt{L^{2}+B^{2}+Z^{2}}}+\frac{L \cdot B \cdot z}{\sqrt{L^{2}+E^{2}+E^{2}}} \times\left(\frac{1}{L^{2}+Z^{2}}+\frac{1}{B^{2}+E^{2}}\right)\right]$.

Using the formula (10) one can calculate stress induced in subsoil under any point of foundation, including characteristic point mentioned above or foundation centre point. The similar formula as the formula (10) is introduced e.g. also in [5].

When calculating foundation settlement by the STN, subsoil is divided into layers. The final settlement (compression) of subsoil under examined point can be calculated using the formula:

$s=\sum_{\mathrm{i}=1}^{n} \frac{\sigma_{x, i}-m_{u} \sigma_{\text {or }, i}}{E_{\text {oxdi }}} \cdot h_{\mathrm{i}}$,

where $s$ is the settlement of examined point (e.g in $\mathrm{mm}), \sigma_{z, i}$ is the vertical component of stress under examined point from overloading by structure $\sigma_{\text {oll }}$ in the centre of i-layer (e.g. in $\mathrm{kPa}$ ), $m_{\mathrm{i}}$ is 
the corrected coefficient of overloading which can be determined for i-layer on the basic of the kind of soil by the Table 1 in the STN (values of $m_{\mathrm{i}}$ are $0.1,0.2$ or 0.3 ), $\sigma_{\text {or }{ }_{j i} \mathrm{i}}$ is the origin effective geostatic stress in the centre of $\mathrm{i}$-layer (e.g. in $\mathrm{kPa}$ ), $h_{\mathrm{i}}$ is the thickness of $\mathrm{i}$-layer (e.g. in $\mathrm{m}$ ), $E_{\text {oed, } \mathrm{i}}$ is the design oedometric modulus of $\mathrm{i}$-layer of subsoil (e.g. in $\mathrm{kPa}$ ).

The relationship between deformation modulus $E_{\text {def }}$ and oedometric modulus $E_{\text {ord }}$ is roughly defined by the formula:

$E_{\text {osd }}=\frac{1}{\beta} \cdot E_{\text {def }}$,

$\beta=1-\frac{2 . v^{2}}{1-v^{2}}$

where $v$ is Poisson's ratio.

As one can see in the formula (11), the vertical stress $\sigma_{z}$ of every layer is reduced on its effective component $\sigma_{z}-m_{*} \sigma_{\text {or }}$, which causes deformation. For the soils, the value $m_{.} \sigma_{o r}$ presents the structural strength which is resistant to deformation. The more small value of $m$, the deformation behaviour of soils is closer to the behaviour of linear elastic material. By the reduction of vertical stress to its effective value, the calculation of settlement in deep direction will be limited to real thickness of deformation zone (the deformation zone under foundation is limited space in subsoil in which we cannot neglect deformation raised by loading from structure). The thickness of layers $h_{\mathrm{i}}$ comforts the variability of design values of deformation characteristics of subsoil and the course of vertical stress (therefore the borders of layers for settlement calculation are chosen in the places of soils interface, in the place of ground water level etc.). When calculating the foundation settlement, it is necessary to consider the influence of stress induced from adjacent buildings or other loading as well as the influence of given foundation on possible settlement of adjacent buildings foundations. The formula (11) is used to calculate settlement not only at various points of foundation base but also at points out from it. The average settlement $s_{m}$ of stiff foundation is the settlement arising from average overloading on foundation base $\sigma_{o l}$. The average settlement $s_{m}$ of flexible foundation is the arithmetic average of calculated values of settlements of representative points on foundation structure. Non-uniform settlement is verified in the range of objects directly related (e.g. inside of parts separated by construction expansion joints). The STN recognizes the following types of non-uniform settlements: relative deflection $\Delta s / L_{T}$; angle deformation $\Delta s / L$ and inclination $\Delta s / b$. In accordance with the requirements of serviceability limits state of building structures, it is necessary that average value of final settlement $s_{m}$ and non-uniform settlement stay in the range quoted in the STN.

\section{Determination of stress in subsoil by the PN}

By the PN, when calculating the stress induced from loading by a structure, the structure is considered to be flexible. Pad foundation under single column and strip foundation under load-bearing wall is considered to be stiff. By the PN, when calculating the foundation settlement, the subsoil is divided also into layers. However, the PN considers the settlement of a layer as a sum of two kinds of settlements. The first one is so called "secondary settlement" $s_{i=}^{s x}$, caused by secondary stress $\sigma_{z s}$ and the second one is so called "primary settlement" $s_{i}$, caused by an additional stress $\sigma_{z a d}$. The secondary stress induced in the subsoil from the reason that at the first stage, the subsoil is loaded by original effective geostatic stress; after an excavation is carried out, the subsoil is relieved by relieving stress $\bar{\sigma}_{z \rho}$ and after construction of structure starts, the subsoil is loading again by stress induced from structure load $\sigma_{z q}$. In the case when the stress induced from structure load is smaller than relieving stress, there is only secondary stress acting in the subsoil. In the case when stress induced from structure load is larger than relieving stress, there are secondary stress acting in subsoil (equal to relieving stress) and also additional stress acting in subsoil (equal to difference between stress induced from structure load and relieving stress). The PN applies following equations:

$$
\begin{aligned}
& \sigma_{z g}=\sigma_{z q} \text { and } \sigma_{z d}=0 \text { when } \sigma_{z q} \leq \bar{\sigma}_{z \rho}, \\
& \sigma_{z s}=\bar{\sigma}_{z \rho} \text { and } \sigma_{z d}=\sigma_{z q}-\bar{\sigma}_{z \rho} \text { when } \sigma_{z q}>\bar{\sigma}_{z \rho} .
\end{aligned}
$$


The PN introduces the coefficient $\eta_{n}$ for calculation of vertical stress $\sigma_{z}$ in the depth $z$ under the corner of the rectangle foundation with the width $B$ and length $L$, which can be calculated using the formula:

$\eta_{n}=\frac{1}{2 a x} \cdot\left\{\operatorname{arctg} \frac{\frac{L}{B}}{\frac{I}{B} \cdot \sqrt{1+\left(\frac{L}{B}\right)^{2}+\left(\frac{x}{B}\right)^{2}}}+\frac{\frac{L}{B} \cdot \frac{x}{B}}{\sqrt{1+\left(\frac{L}{B}\right)^{2}+\left(\frac{x}{B}\right)^{2}}} \cdot\left[\frac{1}{1+\left(\frac{x}{B}\right)^{2}}+\frac{1}{\left(\frac{L}{B}\right)^{2}+\left(\frac{x}{G}\right)^{2}}\right]\right\}$,

Then the vertical stress $\sigma_{z}$ can be calculated using the formula:

$\sigma_{z}=\eta_{n} \cdot q^{\prime}$,

where $q$ is the uniform loading acting on the rectangle with the width B and length $L$. The formula (16) can be used to calculate relieving stress $\bar{\sigma}_{z \rho}$.

The PN introduces the coefficient $\eta_{s}$ for calculation of average stress $\sigma_{z}$ in the depth $z$ under the rectangle with the width $B$ and length $L$ :

$\eta_{g}=\frac{2}{\pi} \cdot\left\{\operatorname{arctg} \frac{B}{\frac{z}{B} \sqrt{1+\left(\frac{L}{B}\right)^{2}+\left(\frac{z}{B}\right)^{2}}}-\frac{\frac{z}{E}}{\frac{E}{B}} \cdot\left[\sqrt{1+\left(\frac{z}{B}\right)^{2}}+\sqrt{\left(\frac{L}{B}\right)^{2}+\left(\frac{z}{B}\right)^{2}}-\sqrt{1+\left(\frac{L}{B}\right)^{2}+\left(\frac{z}{B}\right)^{2}}-\frac{z}{B}\right]\right\}$,

and states that formula (18) can be used to calculate stress under absolute stiff foundation. It means that:

$\sigma_{z}=\eta_{S^{x}} \frac{Q}{B \cdot L}$

where $Q$ is the vertical force acting on foundation.

By the PN, the settlement $s_{i \mathrm{i}}$ of a subsoil layer of thickness $h_{\mathrm{i}}$ can be calculated using formulas:

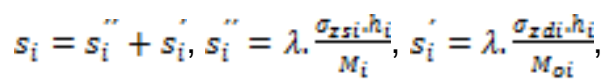

where $s_{i}$ is secondary settlement and $s_{i}$ is primary settlement. The $\lambda$ is a coefficient taking into account a degree of relieving subsoil after excavation is carried out. In the case when construction time (from beginning of excavation to the end of raft structure, including installation of equipment representing permanent load) is less than 1 year, the value of $\lambda$ is null. In the case when mentioned time is more than 1 year, the value of $\lambda$ is $1 . M_{\mathrm{i}}$ and $M_{\text {oi }}$ are secondary and primary oedometric modulus of the soil of the layer $i$. We would like to note that by the PN, deformation zone is limited to the depth where additional stress $\sigma_{z d}$ is smaller than $0.3 . \sigma_{\circ y, i}\left(\sigma_{o r, i}^{\prime i}\right.$ is the effective origin geostatic stress). Concerning the requirements of serviceability limits state of building structures, the PN also prescribes limited values that average value of final settlement $s_{m}$ and non-uniform settlement should not exceed. In comparison with the STN, there are fewer categories of the structures (only 4 categories) and the categories are not compatible so it is very hard to compare the STN and PN. So, e. g. by the PN, there is a category of "industrial halls", for which the average final settlement should not exceed $5 \mathrm{~cm}$ and limited angle deformation $\Delta \mathrm{s} / \mathrm{L}$ is 0.003 . For other categories, there is no prescribed limited angle deformation. The maximal value of average final settlement by the $\mathrm{PN}$ is $15 \mathrm{~cm}$ and it is applied for slender buildings of height more than $100 \mathrm{~m}$.

\section{Example}

To illustrate the consequences of various approaches in determination of stresses induced in subsoil by the STN and the PN, we will introduce an example of a pad foundation, posted in [6], see Fig. 1. The subsoil consists of medium sand (from terrain surface to the depth $2.5 \mathrm{~m}$ ), sandy silt (from depth $2.5 \mathrm{~m}$ to $3.5 \mathrm{~m}$ ), which is followed by medium sand. Underground water level is in the depth of $1.75 \mathrm{~m}$ under terrain surface. The unit weight of medium sand above underground water level is $17.7 \mathrm{kN} . \mathrm{m}^{-3}$, under underground water level is $17.9 \mathrm{kN} . \mathrm{m}^{-3}$. The unit weight of sandy silt is $20.3 \mathrm{kN} . \mathrm{m}^{-3}$. The primary oedometric modulus of medium sand is $\mathrm{M}_{0}=130 \mathrm{MPa}$ and secondary oedometric 
modulus of medium sand is $\mathrm{M}=144 \mathrm{MPa}$. The primary oedometric modulus of sandy silt is $\mathrm{M}_{0}=4.14 \mathrm{MPa}$ and secondary oedometric modulus of medium sand is $\mathrm{M}=7.28 \mathrm{MPa}$. The pad has square shape with $B=L=2.2 \mathrm{~m}$. Vertical loading $N_{n}=950 \mathrm{kN}$ acts centrically. Depth of foundation is $1.0 \mathrm{~m}$.

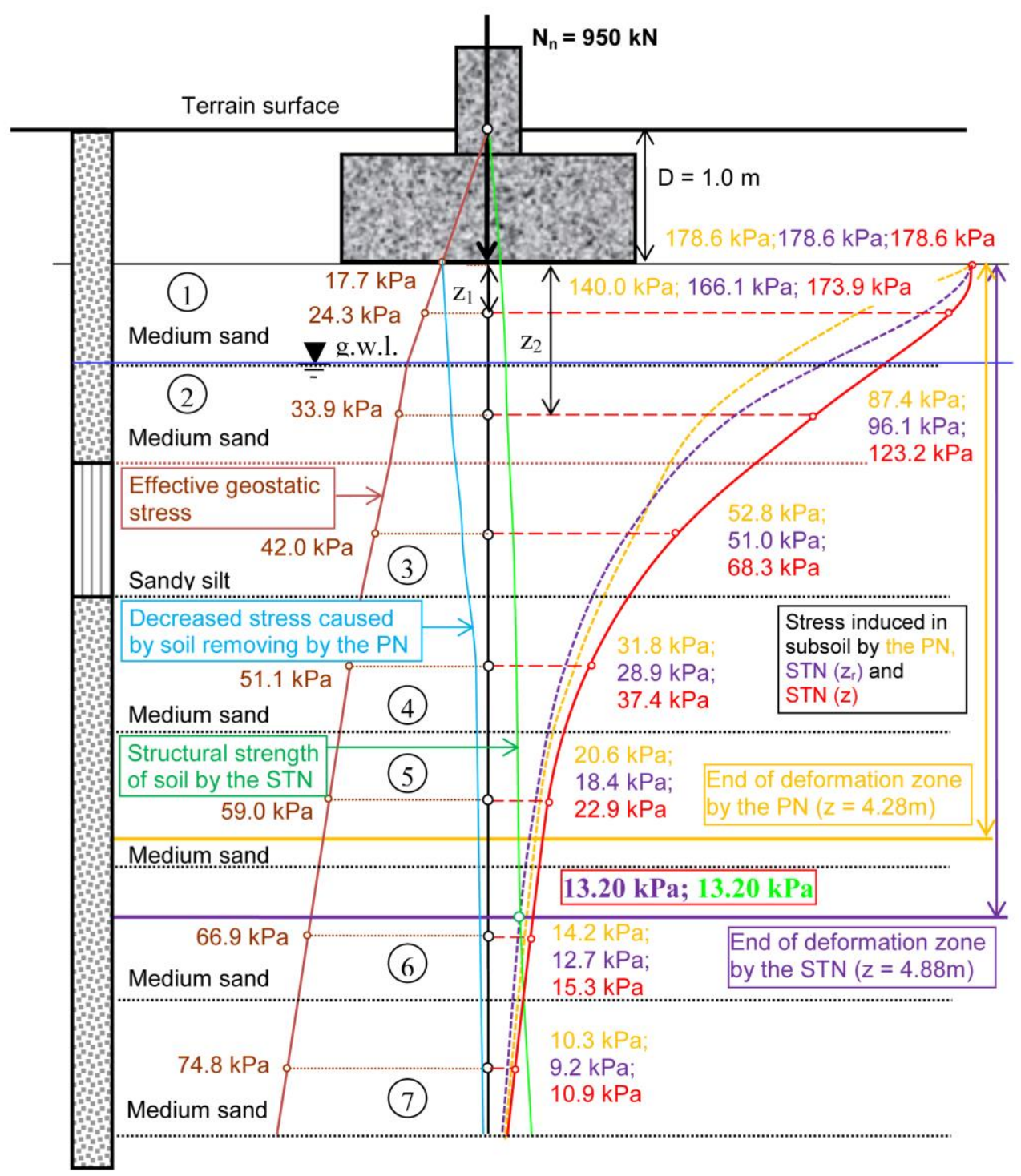

Fig. 1: The course of various stresses induced in pad foundation subsoil.

With the comparison purpose, we will examine only stresses induced in points under foundation centre point. In the Fig. 1 we can see course of various stresses induced in the subsoil in the centre points of subsoil layers, numbered from 1 to 7 . The thickness of the layers $h_{\mathrm{i}}$ and distances $z_{\mathrm{i}}$ from the centre of layer $i$ to foundation base can be seen in the Table 1.

By the STN, the following stresses should be determined in the subsoil: effective geostatic stress $\sigma_{\text {or } i \mathrm{i}}$ (see brown colour line in the Fig. 1 and also in the Table 1); soil structural strength (see green colour line in the Fig. 1 and also in the Table 1) and vertical stress from structure load $\sigma_{z i}(z 1 r i)$ calculated in the depth $z_{r 1 \mathrm{i}}$ but considered to act in the depth $z_{\mathrm{i}}$ (see purple colour line in the Fig. 1 and also in the Table 1). For the comparison purpose, the vertical stress from structure load $\sigma_{z i \text { (ai })}$, calculated in the depth $z_{\mathrm{i}}$ considered to act in the depth $z_{\mathrm{i}}$ is al so introduced (see red colour line in the Fig. 1 and also in the Table 1). 
Table 1: Values of stresses induced in subsoil and differences in vertical stresses by the STN.

\begin{tabular}{|c|c|c|c|c|c|c|c|c|c|}
\hline $\begin{array}{l}\text { Nr. of } \\
\text { layer }\end{array}$ & $\begin{array}{l}\text { Thick. of } \\
\text { layer } \\
h_{\tilde{4}} \\
(\mathrm{~m})\end{array}$ & $\begin{array}{l}\text { Effective } \\
\text { soil unit } \\
\text { weight } \\
\left(\mathrm{kN} \cdot \mathrm{m}^{-3}\right)\end{array}$ & $\begin{array}{c}z_{i} \\
(\mathrm{~m})\end{array}$ & $\begin{array}{c}\text { Effective } \\
\text { geostatic } \\
\text { stress } \\
\square \sigma_{\text {erri }}^{-} \\
(\mathrm{kPa})\end{array}$ & $\begin{array}{c}\text { Soil } \\
\text { structural } \\
\text { strength } \\
\text { m. } \sigma_{\text {ersi }}^{-} \\
(\mathrm{kPa})\end{array}$ & $\begin{array}{c}\text { Vertical } \\
\text { stress } \\
\sigma_{x i[\mathrm{ritri}]} \\
(\mathrm{kPa})\end{array}$ & $\begin{array}{c}\text { Vertical } \\
\text { stress } \\
\sigma_{x i\left[x_{i j}\right.} \\
(\mathrm{kPa})\end{array}$ & 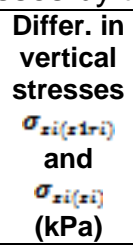 & $\begin{array}{c}\text { Differ. in } \\
\text { vertical } \\
\text { stresses } \\
\sigma_{x i[x 1 r i]} \\
\text { and } \\
\sigma_{x i\left[x_{1}\right.} \\
(\%) \\
\end{array}$ \\
\hline 0 & 0 & & 0 & 17.7 & 3.5 & 178.6 & 178.6 & 0 & 0 \\
\hline 1 & 0.75 & 17.7 & 0.37 & 24.3 & 4.8 & 166.1 & 173.9 & 7.8 & 4.7 \\
\hline 2 & 0.75 & 7.9 & 1.12 & 33.9 & 6.7 & 96.1 & 123.2 & 27.1 & 28.2 \\
\hline 3 & 1 & 10.3 & 2 & 42.0 & 8.4 & 51.0 & 68.3 & 17.3 & 33.9 \\
\hline 4 & 1 & 7.9 & 3 & 51.1 & 10.2 & 28.9 & 37.4 & 8.5 & 29.4 \\
\hline 5 & 1 & 7.9 & 4 & 59.0 & 11.8 & 18.4 & 22.9 & 4.5 & 24.5 \\
\hline 6 & 1 & 7.9 & 5 & 66.9 & 13.4 & 12.7 & 15.3 & 2.6 & 20.5 \\
\hline 7 & 1 & 7.9 & 6 & 74.8 & 14.9 & 9.2 & 10.9 & 1.7 & 18.5 \\
\hline
\end{tabular}

By the PN, the following stresses should be determined in the subsoil: effective geostatic stress $\sigma_{o r}, i$ (see brown colour line in the Fig. 1 and also in the Table 2); decreased stress by soil removing $\bar{\sigma}_{z \rho}$ (see blue colour line in the Fig. 1 and also in the Table 2); vertical stress induced from structure load $\sigma_{z q}$ (from the reason of figure visibility not introduce in the Fig. 1, see the Table 2) and additional vertical stress $\sigma_{z d}$ (see yellow colour line in the Fig. 1 and also in the Table 2).

Table 2: Values of stresses induced in the subsoil by the PN and differences in vertical stresses by

\begin{tabular}{|c|c|c|c|c|c|c|c|}
\hline $\begin{array}{l}\text { Nr. of } \\
\text { layer }\end{array}$ & $\begin{array}{c}\mathrm{z}_{\mathrm{i}} \\
\text { (m) }\end{array}$ & $\begin{array}{c}\text { Effective } \\
\text { geostatic } \\
\text { stress } \sigma_{\text {exi }} \\
(\mathrm{kPa})\end{array}$ & $\begin{array}{c}\text { Decreased } \\
\text { stress by } \\
\text { soil } \\
\text { removing } \\
\bar{\sigma}_{x p}(\mathrm{kPa})\end{array}$ & $\begin{array}{l}\text { Vertical } \\
\text { stress from } \\
\text { structure } \\
\sigma_{\mathrm{xq}}(\mathrm{kPa})\end{array}$ & $\begin{array}{l}\text { Additional } \\
\text { vertical } \\
\text { stress } \\
\sigma_{x d} \\
(\mathrm{kPa})\end{array}$ & 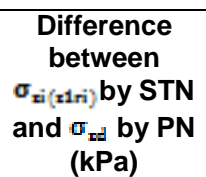 & $\begin{array}{c}\text { Difference } \\
\text { between } \\
\sigma_{x i[\text { inting }} \text { by STN } \\
\text { and } \sigma_{x d} \text { by PN } \\
(\%)\end{array}$ \\
\hline 0 & 0 & 17.7 & 17.7 & 196.3 & 178.6 & 0.0 & 0.0 \\
\hline 1 & $\begin{array}{c}0.37 \\
5\end{array}$ & 24.3 & 17.3 & 157.3 & 140.0 & 26.1 & 18.6 \\
\hline 2 & $\begin{array}{c}1.12 \\
5\end{array}$ & 33.9 & 12.2 & 99.6 & 87.4 & 8.7 & 10.0 \\
\hline 3 & 2 & 42.0 & 6.8 & 59.6 & 52.8 & -1.8 & -3.4 \\
\hline 4 & 3 & 51.1 & 3.7 & 35.5 & 31.8 & -2.9 & -9.1 \\
\hline 5 & 4 & 59.0 & 2.2 & 22.8 & 20.6 & -2.2 & -10.7 \\
\hline 6 & 5 & 66.9 & 1.5 & 15.7 & 14.2 & -1.5 & -10.6 \\
\hline 7 & 6 & 74.8 & 1.0 & 11.3 & 10.3 & -1.1 & -10.7 \\
\hline
\end{tabular}

Concerning the STN, as we can see in the Table 1, the differences between vertical stress from structure load $\sigma_{z i(z x 1 i)}$ calculated in the depth $z_{r 1 i}$ but considered to act in the depth $z_{i \mathrm{i}}$ (see purple colour line in the Fig. 1 and also in the Table 1) and vertical stress from structure load $\sigma_{z i \text { (zi) }}$, calculated in the depth $z_{\mathrm{i}}$ considered to act in the depth $z_{\mathrm{i}}$ (not taking into account influence of foundation depth - see chapter 2 and red colour line in the Fig. 1 and also in the Table 1), is not negligible. So, e. g. in the depth $\mathrm{z}=2 \mathrm{~m}$ (under foundation base), the difference is up to $17.3 \mathrm{kPa}$ (33.9\%). We would like to note that PN does not take into account influence of foundation depth by this way.

The comparison between additional vertical stress from structure calculated by the STN and the PN is introduced in the Table 2. As one can see, in smaller depth, additional vertical stress from the structure calculated by the STN has higher values as that one calculated by the PN but in larger depth, additional stress from the structure calculated by the PN is higher (see also yellow and purple colour lines in the Fig. 1). The difference is not negligible and in the depth $z=0.375 \mathrm{~m}$ (under 
foundation base) it reaches a value of $26.1 \mathrm{kPa}(18.6 \%)$. There is also a difference in determination of the end of deformation zone by the STN and the PN. By the STN, the end of the deformation zone lays in the depth, where additional vertical stress from structure $\sigma_{z i l a 1 r i b}$ is equal to soil structural strength which depends on the kind of soil (see the point where purple and green colour lines meet in the Fig. 1). By the PN, the end of the deformation zone lays in the depth, where additional vertical stress from structure $\sigma_{z d}$ is equal to $30 \%$ of effective geostatic stress $\sigma_{\text {or }, i}$. Applying such rules, the end of deformation zone by the STN lays in the depth of $4.88 \mathrm{~m}$ under the foundation base, where additional vertical stress from structure has a value of $13.2 \mathrm{kPa}$ (see horizontal purple colour line) and by the PN, it lays in the depth of $4.28 \mathrm{~m}$ under the foundation base, where additional vertical stress from structure has a value of $18.4 \mathrm{kPa}$ (see horizontal yellow colour line, the number $18.4 \mathrm{kPa}$ is not seen in the Fig. 1 because of figure visibility).

Concerning the settlement, the value of settlement calculated by the STN is $16.4 \mathrm{~mm}$ (not taking the influence of foundation depth into account) and $11.9 \mathrm{~m}$ (taking the influence of foundation depth into account). The value of settlement calculated by the PN is $15.5 \mathrm{~mm}(1.1 \mathrm{~mm}$ of secondary settlement $+14.4 \mathrm{~mm}$ of primary settlement). The values of settlements are introduced with tenth of $\mathrm{mm}$ for analysis only (mainly for secondary settlement). The relative lower values of settlements calculated by the STN in spite of higher values of stress induced in the subsoil from structures is caused by soil structural strength, see formula (11). However, we would like to note that from the reason that there are many differences in determination of soils shear strength parameters and deformation characteristics in Slovakia and Poland, see [7] and also differences posted in previous chapters, mentioned values of settlement are only informative a cannot be used in comparison and for making conclusions.

\section{Conclusions}

The determination of stresses induced in spread foundation subsoil is very important since one needs to know them to design spread foundation. Stresses induced in spread foundation subsoil depend on many factors, such as foundation stiffness, soil unloading by excavation and soil secondary loading by structure load, influence of foundation depth or of practically incompressible layer etc. Various approaches in determining stresses induced in spread foundation subsoil bring final values, which are quite different. Various values of stresses induced in spread foundation subsoil define also various depths of deformation zone under foundation which influences the values of foundation settlement.

By the author's knowledge, there are many differences in designing geotechnical structures between Slovakia and Poland. The differences are not only in determination of stresses in subsoil as in this case but e. g. also in determination of geotechnical parameters. So applying Standards of one country in designing geotechnical structures in another country without experience and understanding contexts can lead to mistakes. Therefore, harmonization of geotechnical design in Europe will be very helpful.

\section{Acknowledgement}

The research is supported by the European Regional Development Fund and the Slovak state budget for the project "Research Centre of University of Žilina”, ITMS 26220220183.

\section{References}

[1] VERRUIJT, A.: Soil Mechanics. Delft : Delft University of Technology. 315 p. 2006.

[2] ŠIMEK, J. et al.: Soil Mechanics (in Czech language). Prague : SNTL, 388 p. ISBN 80-03-00428-4, 1990.

[3] STN 73 1001. Geotechnical structures. Foundation (in Slovak language), Bratislava : Slovak Standards Institute, 2010.

[4] PN-81/B-03020: Building soils. Foundation bases. Static calculation and design (in Polish language). Warsaw : Polish Committee for Standardization, 1981.

[5] CHAU, N. A.: Foundation of structures (in Vietnamese), $2^{\text {nd }}$ ed., Hanoi : Construction Publishing House, 397 p., 2009. 
[6] OBRYCKI, M - PISARCZYK, S.: Collection of examples from Soil Mechanics. Warsaw : Warsaw University of Technology. 148 p., ISBN 978-83-7207-693-9, 2007.

[7] NGUYEN, G.: Differences in determination of geotechnical parameters in Slovakia and Poland. In: SGEM 2014 : GeoConference on science and technologies in geology, exploration and mining : $14^{\text {th }}$ international multidisciplinary scientific geoconference : conference proceedings: 17-26 June 2014, Albena, Bulgaria. - Sofia: STEF92 Technology, 2014. ISBN 978-619-7105-08-7. p. 299-306. 